\title{
Changes in Dynamic Plantar Pressure for Children with Tarsal Coalition as Compared to Normal Children
}

\author{
Brian Curtin, DO*, Xue-Cheng Liu, MD, PhD, Roger Lyon, MD and Scott Van Valin, MD \\ Department of Orthopaedic Surgery, Children's Hospital of Wisconsin, Medical College of Wisconsin, USA
}

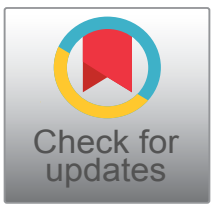

*Corresponding author: Brian Curtin, DO, Department of Orthopaedic Surgery, Children's Hospital of Wisconsin, Medical College of Wisconsin, Milwaukee Campus, 8915 W Connell Ct, Milwaukee, WI 53226, USA

\begin{abstract}
Objectives: One of effective therapeutic treatment involves the use of inserts to alter the foot biomechanical alignment and reduce the pain. It is essential to select an appropriate insert based upon foot functions by dynamic plantar pressure measurements. The goal of this study was to evaluate children's kinetic abnormalities in feet with tarsal coalition using plantar pressure measurements.
\end{abstract}

Design: The design of this study was a prospective cohort with normal control, which is considered as Level 3 Evidence.

Setting: The study was conducted in a motion analysis laboratory at a private pediatric health care system with an academic affiliation.

Patients: Nine patients, ages 6 to 16 years who were diagnosed with tarsal coalition were recruited. Exclusion criteria included the presence of neuromuscular disease or the inability to ambulate.

Methods: Plantar pressure measurements were performed using the EMED platform. Each subject stepped on the platform three times while walking at a self-selected speed along a 5-meter walkway. There were also normal plantar pressure data in 68 typically developing children from a previous study. Four pressure parameters were then calculated in eight anatomical regions, including contact area, peak pressure, pressure-time, and instant of peak pressure.

Main outcome measurements: An independent student t-test and Bonferroni correction was performed to compare dynamic plantar pressure metrics between those of our tarsal coalition subjects and those from previously recorded normal data.

Results: Increased contact area at the midfoot and increased peak pressures at the midfoot, 1st metatarsal and hallux were significant in children with tarsal coalition $(P<0.05)$.
Conclusions: The remarkable kinetic differences between tarsal coalition and normal foot pressure suggest it is important to have plantar pressure analysis in barefoot walking prior to orthotic selection.

\section{Introduction}

Tarsal coalition (TC) is a congenital foot abnormality representing an abnormal connection between two or more tarsal bones. The incidence of tarsal coalition occurs in less than $1 \%$ of the general population [1]. The condition is bilateral in $25 \%-50 \%$ of cases, but does not need to be symptomatic [2]. Up to $75 \%$ of tarsal coalitions may be asymptomatic in patients [3]. Of the symptomatic coalitions, calcaneonavicular $(43.6 \%)$ and talocalcaneal joint (48.1\%), are the most common [4].

Coalitions usually become symptomatic around the ages of 12 to 16 years, which correlates with the time that the coalition begins to ossify. Patients with talocalcaneal typically present in early adolescents (12-16 yr), whereas calcaneonavicular presents from 8 to 12-years-old [1]. Talonavicular and other foot coalitions are rare and usually asymptomatic [5].

Tarsal coalitions are also the most common cause of peroneal spastic flatfoot $[5,6]$. This condition is characterized by peroneal muscle spasms and a painful, rigid valgus deformity of both the forefoot and hindfoot [710]. These are often the presenting complaints from patients with this condition, and these symptoms can be accompanied by pain on the lateral aspect of the calf, tearing of an ossified coalition, and synovial inflammation $[5,10]$. These and adaptive shortening of the peroneal tendons can lead to reflex spasms $[5,10]$. The 
spasms eventually subside, but degenerative arthritis often results from both the deformity and altered intra-articular mechanics [10].

In most children the normal plantar arch develops between the age of 2 and 6 years of age [11]. Children under the age of 6-years-old may have a flexible flat foot, however all but $1 \%$ of the children progress toward a normal plantar arch [12]. Of those that do not develop a normal arch, many will have structural abnormalities such as tarsal coalition. Waseda, et al. further investigated the growth progression of the pediatric plantar arch [13]. Foot length extension began at age 6-years-old for both male and females, but females plateaued in foot length one year earlier than males at age 13 years [13]. Navicular height was also measured and showed that height increased from age 6-13 years old for males and 8-13 years old for females. Lastly, Waseda, et al. calculated the arch height ratio that demonstrated the pediatric plantar arch was essentially flat until age 10-years-old for females and 11-years-old for males [13].

Treatment options range from conservative to surgical. More conservative options include arch supports and medial heel wedges for minor symptoms [14-16]. Casting has also shown to be an effective conservative treatment option, but has failed to provide long-term relief [17].

Symptomatic calcaneonavicular and talocalcaneal coalitions are often treated with surgical management if nonoperative treatment fails to alleviate the patient's symptoms. Operative methods are primarily limited to either excision of the coalition or arthrodesis. Patient age, type and extent of the coalition, and the presence of degenerative changes in the joints affect the choice of procedures. Excision of the calcaneonavicular bar has been reasonably successful in some patients. More than $85 \%$ of patients did not require additional operative procedures after initial resection [3,18-20]. Patients with persistent pain after coalition excision may need a subsequent triple arthrodesis [21,22]. Although the triple arthrodesis is occasionally needed for patients with a talocalcaneal bar, simple resection has yielded good results [23-26].

There are several ways to assess the functional characteristics for tarsal coalition beyond plain radiographs, computerized tomographic, and magnetic resonance imaging. Besides clinical exam, electromyography and plantar pressure platforms can evaluate a foot gait analysis. The use of plantar pressure and gait analysis, allows for a dynamic measurement as compared to standard imaging, which is static. Additionally, with standard imaging there is risk of radiation exposure.

The goal of this study is 1) To characterize the dynamic plantar pressure patterns of children with tarsal coalition; 2) Compare differences of pressure metrics between tarsal coalition and normal children.

\section{Materials and Methods}

Nine patients, ages 6 to 16 years who were diagnosed with tarsal coalition were recruited from clinics with a mean age of 13.6 years. Exclusion criteria included the presence of a lower extremity injury within one year, any history of lower extremity fractures, neuromuscular disease, foot deformities, prior foot surgeries, or an inability to ambulate. Pediatric orthopedic surgeons conducted physical examinations of each subject in the experimental and control group. This study has been approved by the Institutional Review Board and followed the principles of Declaration of Helsinki. All subjects signed a written informed assent and the subject's parent or legal guardian signed a written informed consent on behalf of the minor prior to data collection.

Gait analysis was performed on all subjects by expe-

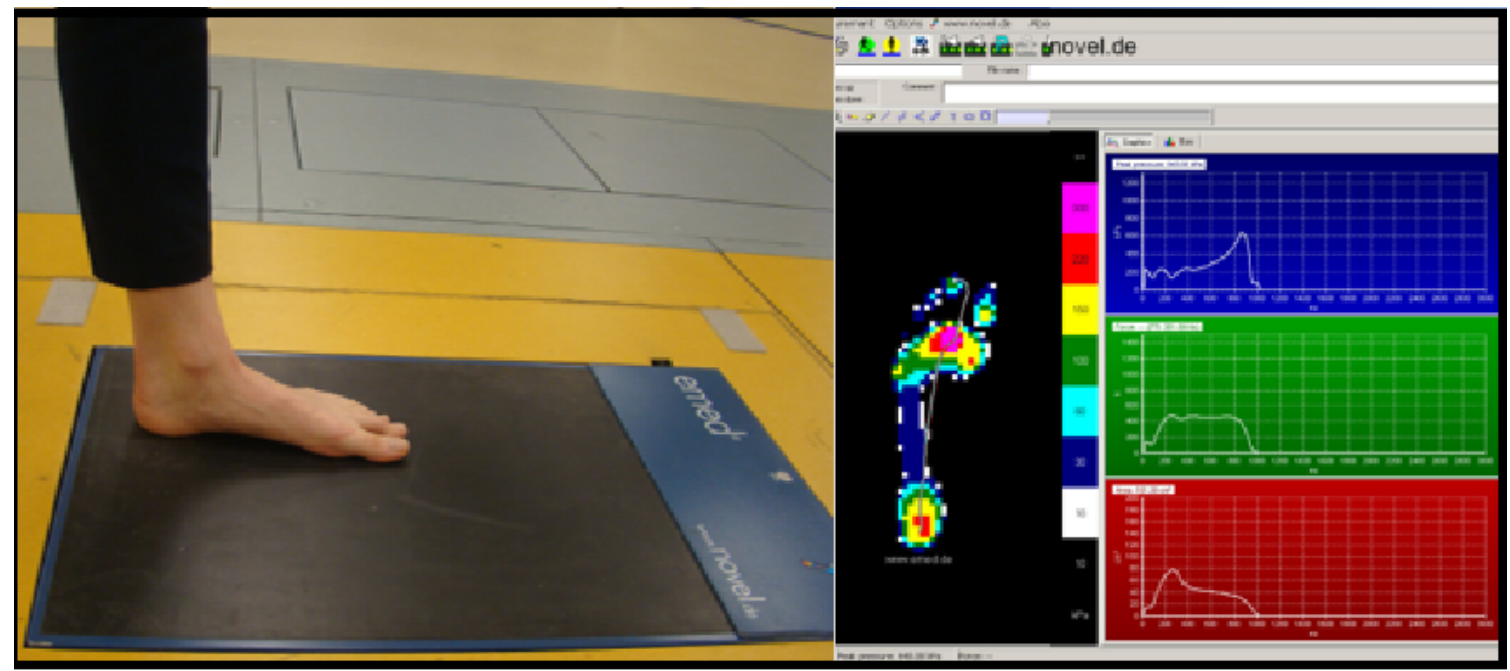

Figure 1: Plantar pressure measurements, including the contact area, peak pressures, and timing during stance phase, were performed using the EMED platform. 
rienced researchers, including physicians and engineers in the center for motion analysis laboratory at a private pediatric health care system with an academic affilia-

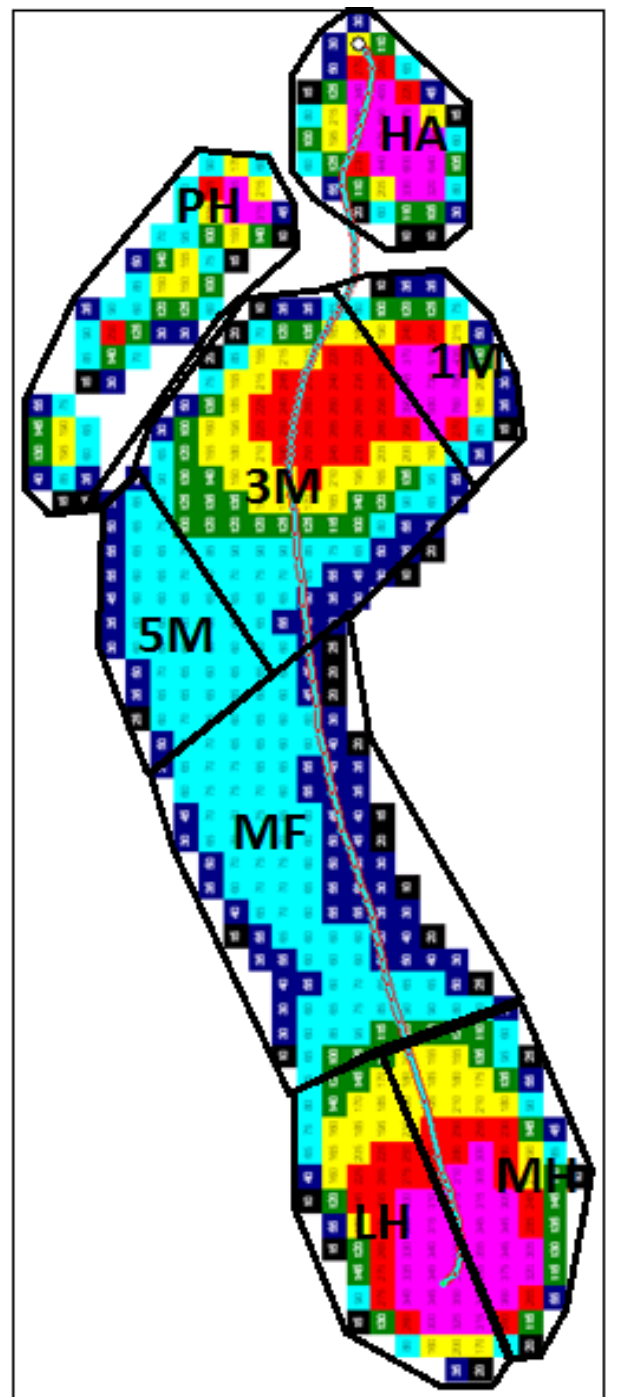

Figure 2: Eight anatomical regions were mapped and defined, including medial and lateral hindfoot, midfoot, $1^{\text {st }}, 3^{\text {rd }}$ and $5^{\text {th }}$ metatarsal, hallux, and phalanges 2 to 5 . The left foot with TC presents an increased contact area on the midfoot and peak pressure on the hallux and $1^{\text {st }}$ metatarsal for this patient. tion. Plantar pressure measurements were performed using the EMED platform system (Novel Inc, St. Paul, MN). The system's sensor plate dimensions are $582 \times$ $340 \pm 20 \mathrm{~mm}$ and consist of a total of 2,736 sensors with a resolution of 4 sensors per $\mathrm{cm}^{2}$ at a frequency of 60 $\mathrm{Hz}$ (frame rate per second). The accuracy of the EMED pressure system is $+/-5 \%$ of mean value [27]. Hughes, et al. found that this system produced excellent reliability for most force, contact area, and pressure variables when the mean of three results was used (Figure 1) [27]. Those variables had reliability coefficients above 0.9.

Each subject stepped on the platform three times while walking at a self-selected speed along a 5-meter walkway. Eight anatomical regions were mapped to determine abnormal pressure distribution: medial hindfoot, lateral hindfoot, midfoot, first metatarsal, third metatarsal, fifth metatarsal, hallux, and phalanges two to five. The boundaries were determined as seen in Figure 2. A total of four pressure parameters were calculated at each of those mapped regions. Pressure parameters consisted of plantar contact area, maximal ground reaction force, peak pressure, and pressure time (loading) (Figure 3).

An independent student t-test and Bonferroni correction were performed to compare EMED metric changes between those of our TC subjects and those from previously recorded normal data. Prior normative data were established by having sixty-six normal children; age 6 to 16-years-old walk along a 5-m walkway at self-selected speeds [28].

\section{Results}

Significant differences in pressure metrics between tarsal coalition subjects and normal are shown in Table 1. Comparison of the contact area between the eight foot regions are illustrated in Figure 4. There were significant differences for contact area of the medial hindfoot, lateral hindfoot, midfoot, fifth metatarsal and first metatarsal when compared to normative data $(P<$ 0.0001 ). The largest contact area is found in the midfoot, followed by the lateral hindfoot.

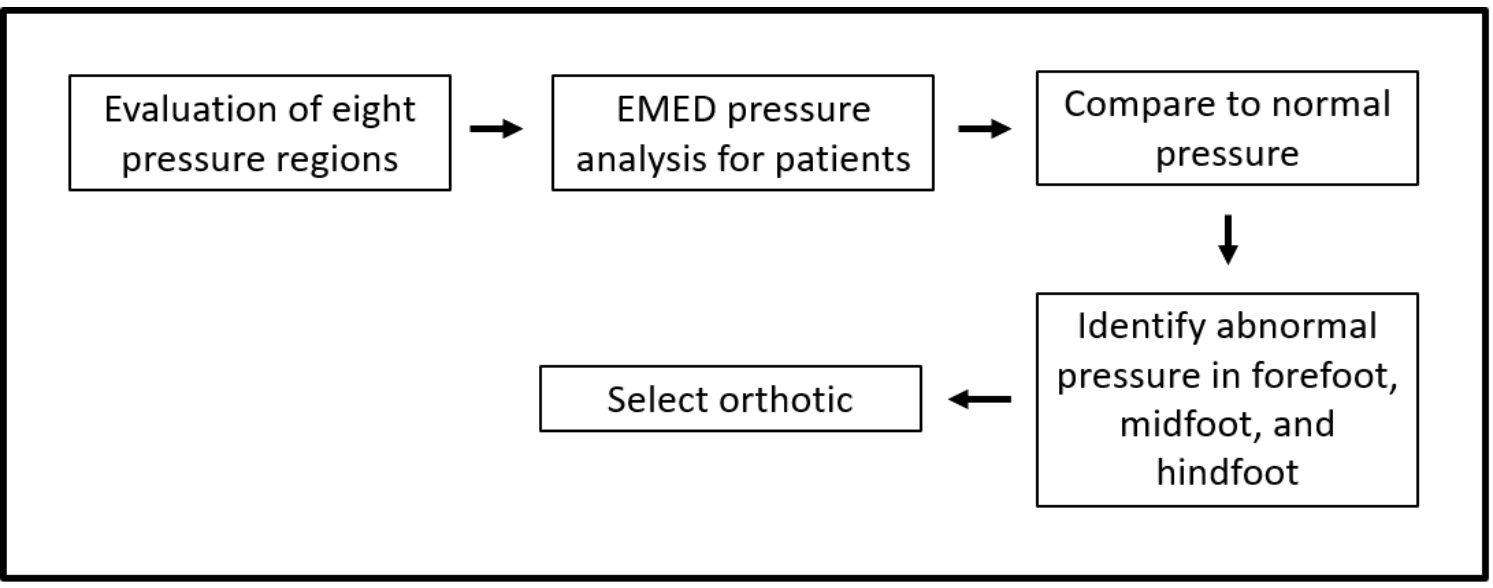

Figure 3: Overview of the process from EMED pressure measurements to foot orthotic selection. 


\section{Contact Area}

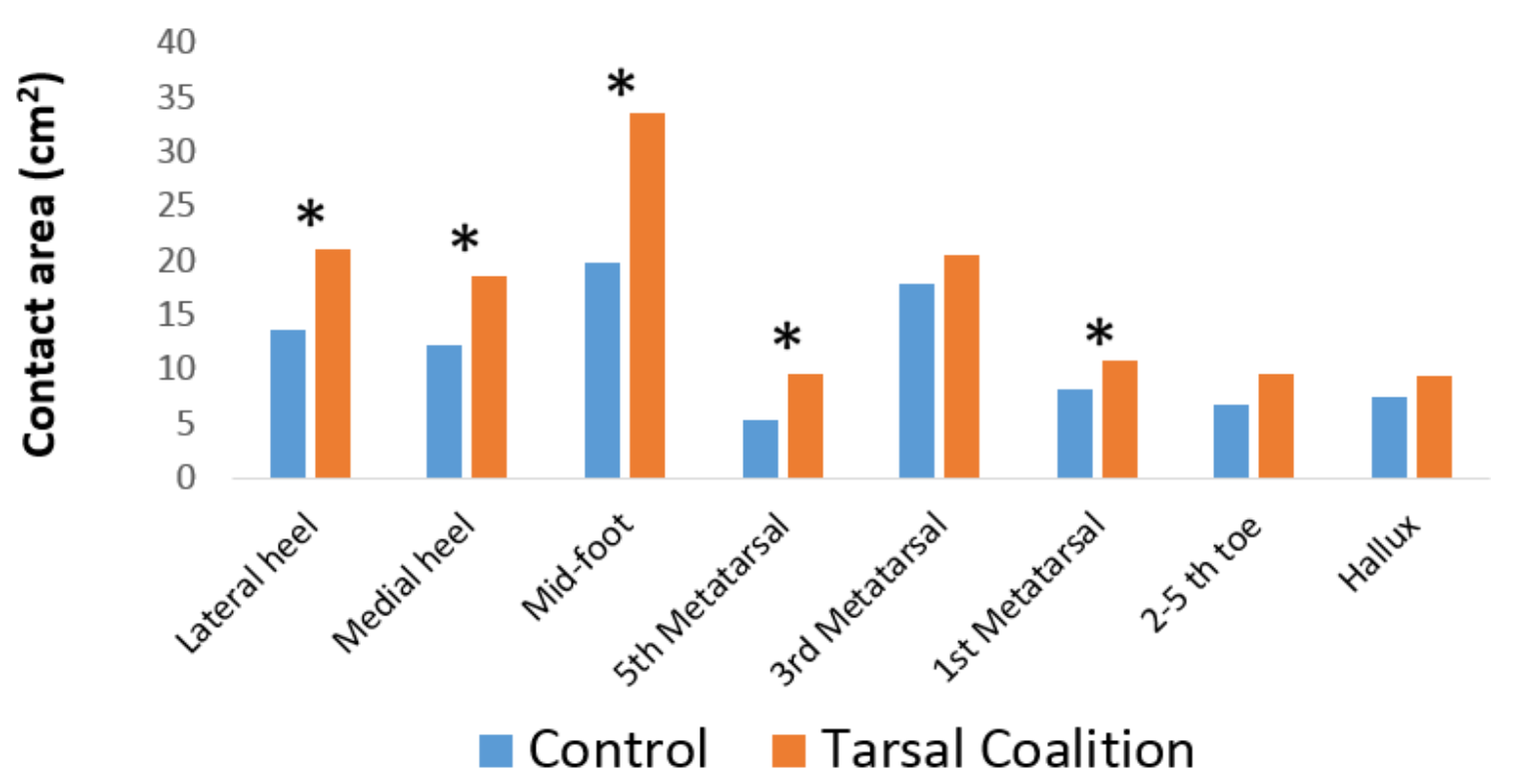

Figure 4: Comparison of the contact area between eight foot regions of subjects with tarsal coalition.

\section{Peak Pressure}

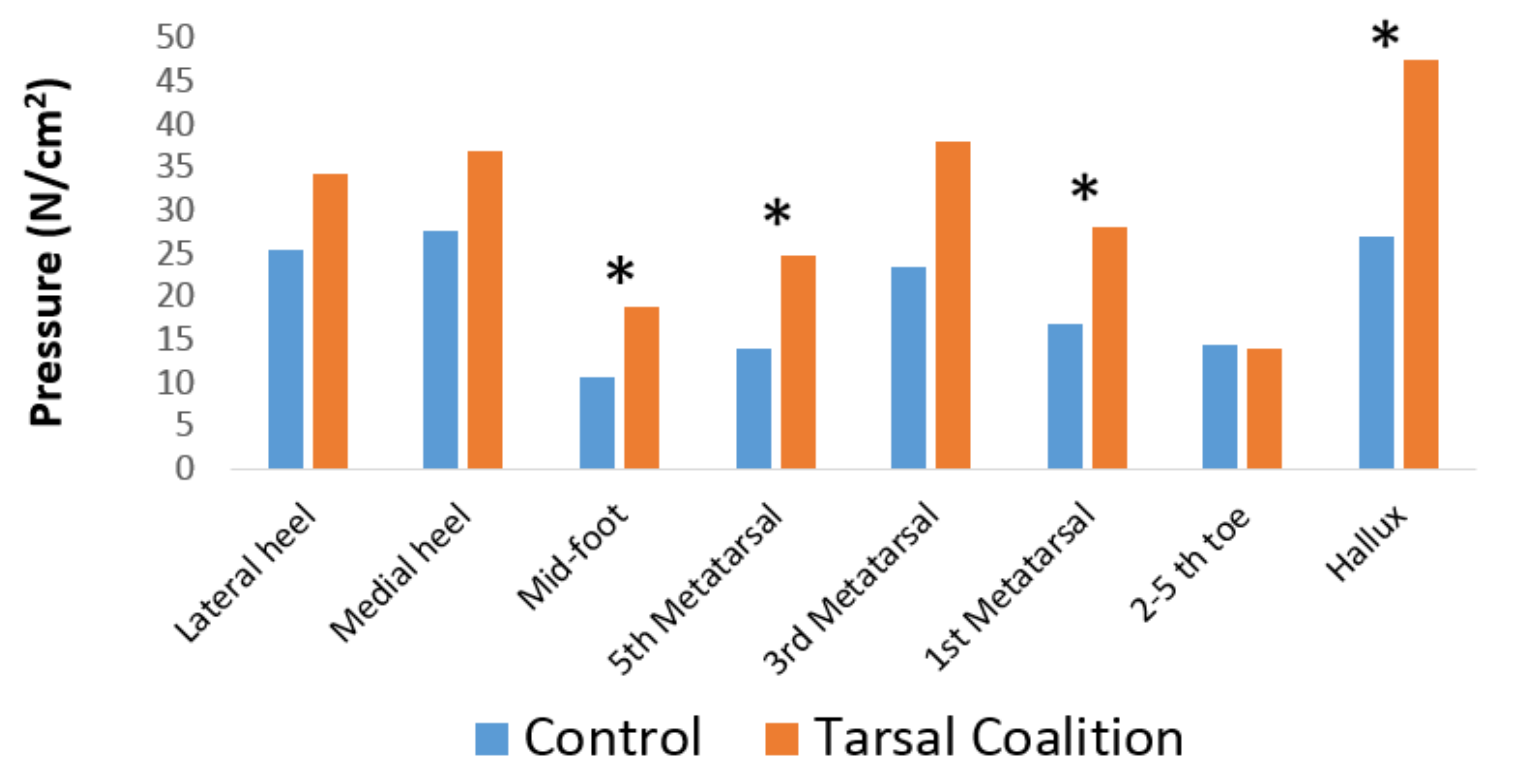

Figure 5: Comparison of the peak pressure between eight foot regions of subjects with tarsal coalition.

Contact time is defined by an occurrence of peak pressure as a percentage of the entire stance phase. The greatest contact time was seen in the third metatarsal head (88\%) and first metatarsal head (84\%). The lateral hindfoot was in contact with the ground the least for $58 \%$ of stance phase.

The highest pressures were generated in the hallux and first metatarsal head. The lowest pressures were found in the second to fifth toes. Significant differences in peak pressures were noted in the hallux, first metatarsal, fifth metatarsal and midfoot. Comparison of the peak pressures between eight foot regions is demonstrated in Figure 5 and Table 1.

The force-time integral reveals the greatest values occurred in the third metatarsal head followed by the medial hindfoot. Loading in all areas of the foot besides the second to fifth toes were significantly different than those of normal children.

Given the results of increased contact area at the midfoot and increased peak pressures at the midfoot, first metatarsal and hallux in children with tarsal coalition, a reduced mid-foot arch and more pronated fore- 
Table 1: Significant differences in pressure metrics between tarsal coalition subjects and normal subjects. (Mean $\pm S D, P<0.05$ ).

\begin{tabular}{|c|c|c|c|}
\hline Parameters & Coalition & Normal & p-value \\
\hline \multicolumn{4}{|c|}{ Contact Area $\left(\mathrm{cm}^{2}\right)$} \\
\hline Lateral-heel & $21.1 \pm 7.3$ & $13.6 \pm 5.7$ & $P<0.0001$ \\
\hline Medial-heel & $18.6 \pm 6.5$ & $12.2 \pm 3.1$ & $P<0.0001$ \\
\hline Mid-foot & $33.6 \pm 14.0$ & $19.8 \pm 10.9$ & $P<0.0001$ \\
\hline $5^{\text {th }}$ Metatarsal & $9.5 \pm 3.1$ & $5.3 \pm 1.5$ & $P=0.0001$ \\
\hline $1^{\text {st }}$ Metatarsal & $10.7 \pm 2.1$ & $8.1 \pm 2.4$ & $P<0.0001$ \\
\hline \multicolumn{4}{|c|}{ Peak Pressure (N/cm²) } \\
\hline Mid-foot & $18.7 \pm 8.2$ & $10.7 \pm 5.5$ & $P<0.0001$ \\
\hline $5^{\text {th }}$ Metatarsal & $24.8 \pm 18.0$ & $14.0 \pm 11.3$ & $P=0.0011$ \\
\hline $1^{\text {st }}$ Metatarsal & $28.0 \pm 10.6$ & $16.8 \pm 9.3$ & $P<0.0001$ \\
\hline Hallux & $47.4 \pm 24.0$ & $27.0 \pm 14.9$ & $P<0.0001$ \\
\hline \multicolumn{4}{|c|}{ Instant of Peak Pressure [\%ROP] } \\
\hline $1^{\text {st }}$ Metatarsal & $82.3 \pm 5.4$ & $70 \pm 14.9$ & $P=0.0001$ \\
\hline Hallux & $88.6 \pm 5.9$ & $81.5 \pm 9.6$ & $P=0.0001$ \\
\hline \multicolumn{4}{|c|}{ Pressure-time Integrals $\left[\left(\mathrm{N} / \mathrm{cm}^{2}\right) \times \mathrm{s}\right]$} \\
\hline Lateral-heel & $9.2 \pm 3.0$ & $5.3 \pm 5.5$ & $P<0.0001$ \\
\hline Medial-heel & $9.8 \pm 3.2$ & $5.8 \pm 5.7$ & $P<0.0001$ \\
\hline Mid-foot & $6.7 \pm 2.7$ & $3.8 \pm 12.3$ & $P<0.0001$ \\
\hline $5^{\text {th }}$ Metatarsal & $7.7 \pm 5.2$ & $4.2 \pm 8.3$ & $P<0.0001$ \\
\hline $3^{\text {rd }}$ Metatarsal & $10.4 \pm 4.4$ & $7.1 \pm 8.6$ & $P=0.0004$ \\
\hline $1^{\text {st }}$ Metatarsal & $8.0 \pm 3.4$ & $5.4 \pm 8.3$ & $P<0.0001$ \\
\hline Hallux & $9.7 \pm 5.2$ & $6.5 \pm 9.1$ & $P=0.0014$ \\
\hline
\end{tabular}

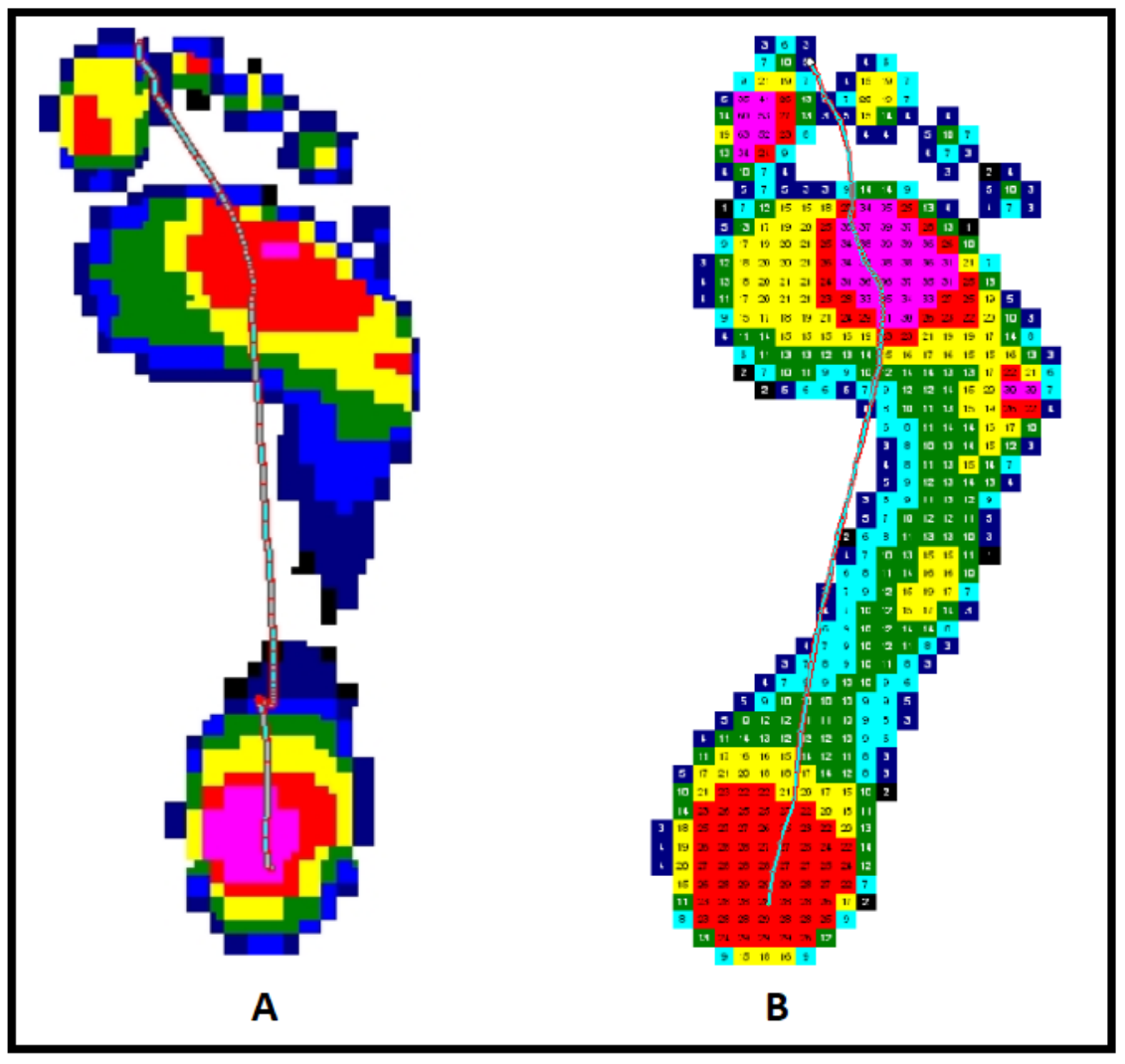

Figure 6: Comparison of plantar pressure distribution between TC and normal: A) A normal pressure distribution of the right foot; B) A 13-year-old girl diagnosed with calcaneonavicular coalition presented with an increased contact area and peak pressure at the midfoot as compared to normal. 
foot were noted $(P<0.0001)$ (Figure 6$)$.

\section{Discussion}

The results of our study provided values of pressure distribution of the pediatric foot with tarsal coalition using the EMED platform system. Children with tarsal coalition revealed significant differences in peak pressure, contact area, and loading when compared with the EMED measurements in normal feet. The midfoot revealed the greatest differences. The midfoot had a significantly higher peak pressure, which was in agreement with Hetsroni, et al. [29]. Although normal pediatric subjects showed lower loading in the medial midfoot [30], our subjects demonstrated significantly greater loading, contact area and peak pressures when compared to normal subjects. In additional study findings of normal pediatric patients, the largest contact area was found in the third metatarsal head, followed by the lateral midfoot and hindfoot. The peak pressures were greatest in the heel and hallux and the lowest in the midfoot under the arch [28].

Tarsal coalition induces malalignments within the subtalar joint. During stance phase of ambulation, the subtalar joint rotates from 4 degrees of external to 6 degrees of internal rotation [31]. With tarsal coalition, lack of external rotation puts strain onto the talonavicular and calcaneocuboid joints. This additional stress on the talonavicular and calcaneocuboid joints leads to a planovalgus deformity. The abnormal bony structures created by a tarsal coalition, leads to increased plantar contact area and loading at the midfoot, as well as at the first metatarsal head and hallux. The combined results may explain that most children in our study had a reduced mid-foot arch and had a pronated forefoot. This further limits the foot segment motions during the first rocker and second rocker, eventually affecting the toe-off at the third rocker, providing reduced power for ambulation.

Given that plantar pressure is a dynamic measurement that is dependent on the ground reaction force, Hetsroni, et al. investigated how tarsal coalition compares to normal subjects while running and walking [29]. The study demonstrated that preoperative feet had significantly higher medial midfoot pressures compared with the control group both during walking and running. Post bar resection, the medial midfoot did not demonstrate significantly higher pressure compared to the control foot while walking. However, during running, this segment's pressures were significantly higher both in preoperative $(p=0.000)$ and in postoperative $(p=$ 0.023 ) feet compared with the control group. This study further supports the use of dynamic plantar pressure measurements for those children with tarsal coalition, both preoperatively and postoperatively. Even postoperatively, children may still demonstrate clinical symptoms of tarsal coalition while running and not walking.
The EMED system allowed patients to walk barefoot at a self-selected speed. Given that the EMED system has patients walk barefoot, it does not take into account the larger contact area that shoes provide, therefore decreasing the peak pressure. Nyska, et al. found that shoes increased the loading of the medial forefoot by nearly $50 \%$, whereas the lateral forefoot's loading decreased by $60 \%$ when compared with the patients who did not wear shoes [32]. He also noted that the force significantly decreased in all other areas of the foot except the medial forefoot in patients who wore shoes. The use of orthotics or inserts may assist in elevation of the mid arch height, therefore reducing midfoot pronation and improve the hindfoot valgus. These corrections will decrease contact area, peak pressure, and loading of the medial midfoot and shift the load to the lateral aspect of the forefoot.

Our findings demonstrated that there are remarkable abnormal dynamic plantar pressures distribution among the hindfoot, midfoot and forefoot for children with tarsal coalition. In such patients, most likely the use of inserts to alleviate the foot pain and restore the arch height has not been successful. Based on the interpretation of the dynamic plantar pressures, a better customized insert may be prescribed to effectively treat the foot deformities. Therefore, the understanding of the abnormal plantar pressure distribution may be a primary step in the evaluation of tarsal coalition or in the determination of the therapeutic or orthotic treatment.

While no known studies have directly investigated the use of orthotics in children with tarsal coalition and how it effects plantar pressure metrics, studies have looked at how orthotics can reduce pain. Powell, et al. compared the efficacy of "custom" made orthotics to those "off the shelf" in children with juvenile arthritis [33]. The custom orthotic group showed significantly greater improvements in overall pain, speed of ambulation, activity limitations, foot pain and level of disability when compared with the "off the shelf" insert [33].

Prior studies of dynamic plantar pressure used vertical or horizontal lines to divide the foot in three or five regions (not using free-mapping) $[30,34,35]$. This method however does not take into account anatomical variations of the foot that may be altered by pronation or supination even in the normal children. Free mapping eight segments as described by Liu, et al. better accommodates for anatomic variants [28]. Thometz, et al. has showed a significant correlation between dynamic pressure measurements and radiographic angles and a presentation of an alignment of the foot [36]. This study however investigated children with residual clubfoot.

The selection age for the children in our study was determined by the fact that the normal plantar arch develops between the age of 2 and 6 years of age [11]. In addition, soft tissue and bone ossification of the 
pediatric foot develops until the age of 5 [37]. Children over the age of 12 with incomplete longitudinal arches may have increased pressure in the midfoot region [38]. Lastly, mature gait appears around 7 years of age $[24,39]$. Limitations of this study include a small sample size of nine children and one follow-up visit. Further studies should delineate differences of pressure parameters of each type of tarsal coalition as compared to age matched normal subjects.

As a conclusion of this study, dynamic plantar pressure results of tarsal coalition feet showed significant differences compared to those of normal feet, showing malalignments of the hind-foot, midfoot and forefoot. The remarkable kinetic differences indicate that those biomechanical abnormalities of the foot impair the walking ability, which is necessary to use a foot orthotic to improve the alignment and function. It is essential to understand those pressure patterns in order to correctly select the orthotics. We recommend every patient to have a plantar pressure analysis during barefoot walking to evaluate the three foot segments and determine the optimal orthotic that reduces the hindfoot valgus, increases the mid-foot arch, and corrects the forefoot supination or pronation.

\section{Acknowledgments}

We would like to thank, Dr. Tarima, Ph.D, Medical College of Wisconsin in the support of statistical analysis. We also appreciate Mr. Marquez-Barrientos, MS, Biomedical engineer, Center for Motion Analysis for subject gait analysis.

\section{References}

1. Cass AD, Camasta CA (2010) A review of tarsal coalition and pes planovalgus: Clinical examination, diagnostic imaging, and surgical planning. J Foot Ankle Surg 49: 274293.

2. Resnick D (1984) Talar ridges, osteophytes, and beaks: A radiologic commentary. Radiology 151: 329-332.

3. Khoshbin A, Bouchard M, Wasserstein D, Leroux $T$, Law PW, et al. (2015) Reoperations after tarsal coalition resection: A Population-based Study. J Foot Ankle Surg 54: 306-310.

4. Kulik SA, Clanton TO (1996) Foot fellow's review: Tarsal coalition. Foot Ankle Int 17: 286-296.

5. Mosier KM, Asher M (1984) Tarsal coalitions and peroneal spastic flatfoot. A review. J Bone Joint Surg Am 66: 976984.

6. Zhou B, Tang K, Hardy M (2014) Talocalcaneal coalition combined with flatfoot in children: Diagnosis and treatment: A review. J Orthop Surg Res 9: 129.

7. Cowell HR (1975) Diagnosis and management of peroneal spastic flatfoot. Instr Course Lect 24: 94-103.

8. Harris RI, Beath T (1955) Etiology of peroneal spastic flat foot. J Bone Joint Surg Am 37: 169-183.

9. Slomann HC (1921) On coalition calcaneo-navicularis. J Orthop Surg 3: 586-602.
10. Knapp HP, Tavakoli M, Levitz SJ, Sobel E (1998) Tarsal coalition in an adult with cavovarus feet. J Am Podiatr Med Assoc 88: 295-300.

11. Volpon JB (1994) Footprint analysis during the growth period. J Pediatr Orthop 14: 83-85.

12. Pfeiffer M, Kotz R, Ledl T, Hauser G, Sluga M (2006) Prevalence of flat foot in preschool-aged children. Pediatrics 118: 634-639.

13. Waseda A, Suda Y, Inokuchi S, Nishiwaki Y, Toyama $Y$ (2014) Standard growth of the foot arch in childhood and adolescence-Derived from the measurement results of 10,155 children. Foot Ankle Surg 20: 208-214.

14. Nalaboff KM, Schweitzer ME (2008) MRI of tarsal coalition: Frequency, distribution, and innovative signs. Bull NYU Hosp Jt Dis 66: 14-21.

15. Cowell HR, Elener V (1983) Rigid painful flatfoot secondary to tarsal coalition. Clin Orthop Relat Res 177: 54-60.

16. Carli A, Leblanc E, Amitai A, Hamdy RC (2014) The evaluation and treatment of pediatric tarsal coalitions. A critical analysis review. JBJS Rev 2: 1-12.

17. Kumar SJ, Guille JT, Lee MS, Couto JC (1992) Osseous and non-osseous coalition of the middle facet of the talocalcaneal joint. J Bone Joint Surg Am 74: 529-535.

18. Scranton PE Jr (1987) Treatment of symptomatic talocalcaneal coalition. J Bone Joint Surg Am 69: 533-539.

19. Mosca VS, Bevan WP (2012) Talocalcaneal tarsal coalitions and the calcaneal lengthening osteotomy: The role of deformity correction. J Bone Joint Surg Am 94: 1584-1594.

20. Luhmann SJ, Schoenecker PL (1998) Symptomatic talocalcaneal coalition resection: Indications and results $\mathrm{J}$ Pediatr Orthop 18: 748-754.

21. Gonzalez P, Kumar JS (1990) Calcaneonavicular coalition treated by resection and interposition of the extensor digitorum brevis muscle. J Bone Joint Surg Am 72: 71-77.

22. Mitchell GP, Gibson JM (1967) Excision of calcaneonavicular bar for painful spasmodic flat foot. J Bone Joint Surg Am 49: 281-287.

23. Comfort TK, Johnson LO (1988) Resection for symptomatic talocalcaneal coalition. J Pediatr Orthop 18: 283-288.

24. Ferrari J, Watkinson D (2005) Foot pressure measurement differences between boys and girls with reference to hallux valgus deformity and hypermobility. Foot Ankle Int 26: 739747.

25. Elkus RA (1986) Tarsal coalition in the young athlete. Am J Sports Med 14: 477-480.

26. Wilde PH, Torode IP, Dickens DR, Cole WG (1994) Resection for symptomatic talocalcaneal coalition. J Bone Joint Surg Br 76: 797-801.

27. Hughes J, Clark P, Linge K, Klenerman L (1993) A comparison of two studies of the pressure distribution under the feet of normal subjects using different equipment. Foot Ankle 14: 514-519.

28. Liu XC, Thometz JG, Tassone C, Barker B, Lyon R (2005) Dynamic plantar pressure measurement for the normal subject: Free-mapping model for the analysis of pediatric foot deformities. J Pediatr Orthop 25: 103-106.

29. Hetsroni I, Ayalon M, Mann G, Meyer G, Nyska M (2007) Walking and running plantar pressure analysis before and after resection of tarsal coalition. Foot Ankle Int 28: 575580. 
30. Bowen TR, Miller F, Castagno P, Richards J, Lipton G (1998) A method of dynamic foot-pressure measurement for the evaluation of pediatric orthopedic foot deformities. $J$ Pediatr Orthop 18: 789-793.

31. Wright DG, Desai SM, Henderson WH (1964) Action of the subtalar and ankle-joint complex during the stance phase of walking. J Bone Joint Surg Am 46: 361-382.

32. Nyska M, McCabe C, Linge K, Laing P, Klenerman L (1995) Effect of the shoe on plantar foot pressures. Acta Orthop Scand 66: 53-56.

33. Powell M, Seid M, Szer IS (2005) Efficacy of custom foot orthotics in improving pain and functional status in children with juvenile idiopathic arthritis: A randomized trial. J Rheumatol 32: 943-950.

34. Cooper A, Chhina H, Howren A, Alvarez C (2014) The contralateral foot in children with unilateral clubfoot, is the unaffected side normal? Gait Posture 40: 375-380.
35. Maurer JD, Ward V, Mayson TA, Davies KR, Alvarez CM, et al. (2014) Classification of midfoot break using multisegment foot kinematics and pedobarography. Gait Posture 39: 1-6.

36. Thometz JG, Liu XC, Tassone JC, Klein S (2005) Correlation of foot radiographs with foot function as analyzed by plantar pressure distribution. J Pediatr Orthop 25: 249-252.

37. Beck RJ, Andriachhi TP, Kuo KN, Fermier RW, Galante JO (1981) Changes in the gait patterns of growing children. J Bone Joint Surg Am 63: 1452-1457.

38. Hallemans A, D'Aout K, De Clercq D, Aerts P (2003) Pressure distribution patterns under the feet of new walkers: The first two months of independent walking. Foot Ankle Int 24: 444-453.

39. Bertsch C, Unger H, Winkelmann W, Rosenbaum D (2004) Evaluation of early walking patterns from plantar pressure distribution measurements: First year results of 42 children. Gait Posture 19: 235-242. 